\title{
BLACK HOLE MASS MEASUREMENTS WITH REST-FRAME OPTICAL QUASAR SPECTRA AT $3<\mathbf{z}<6$
}

\author{
Hyunsung David Jun, Myungshin Im, Hyung Mok Lee, and AKARI QSONG team \\ Department of Physics and Astrnomy, Seoul National University, Seoul 151-747, Korea \\ E-mail: hsjun@astro.snu.ac.kr \\ (Received July 01, 2012; Accepted July 27, 2012)
}

\begin{abstract}
We summarize the progress on the rest-frame optical spectroscopy of quasars at $3<\mathrm{z}<6$, from the AKARI QSONG program. QSONG (Quasar Spectroscopic Observations with NIR Grism) is an AKARI space telescope mission program which utilizes the unique spectroscopic capability in the wavelength range of $2.5-5 \mu \mathrm{m}$. This spectral window has been utilized for detecting redshifted $\mathrm{H} \alpha$ emission lines of our high redshift subsample of quasars. From the calculated emission line widths and luminosities we measured supermassive black hole masses using well calibrated optical mass estimators. Science topics regarding optical based black hole masses at high-z are discussed.
\end{abstract}

Key words: galaxies: active; infrared: telescope; conferences: proceedings

\section{INTRODUCTION}

The recent discovery of $10^{10} M_{\odot}$ supermassive black holes (hereafter BHs, McConnell et al., 2011) further constrained the massive limit of BHs, pushing the previous $10^{9} M_{\odot}$ order limit to heavier regimes. The $M_{B H}-\sigma_{*}$ relation, connecting the mass of the $\mathrm{BH}$ and that of its host galaxy bulge, hints that extremely massive BHs are likely to be the most massive systems out of BH-host galaxy co-evolution. This study on the growth of massive BHs however becomes interesting as we still find $10^{9} M_{\odot} \mathrm{BHs}$ in distant active galaxies, even up to $\mathrm{z}=7$ (Mortlock et al., 2011). For BHs to grow into such masses at high redshifts, it would have required rapid mass growth rate in the early universe (Volonteri \& Rees, 2005), thus providing constraints on the $\mathrm{BH}$ evolutionary scenario.

In understanding the nature of distant, growing $\mathrm{BHs}$, reliable mass estimation is necessary. However, $\mathrm{BH}$ masses in active galaxies are from singleepoch mass estimators calibrated with rest-frame optical spectra, having few factors of uncertainty (Onken et al., 2004). In addition to this uncertainty rest-frame UV emission lines have been used to weigh BHs at high- $\mathrm{z}$, where BH mass from some of UV lines have significant discrepancy with that from $\mathrm{H} \beta$ (Shen et al., 2012).

Therefore we introduce a near-infrared grism study of high-z quasars, covering the $2.5-5 \mu \mathrm{m}$ window suitable to observe the redshifted rest-frame optical spectra. The AKARI mission Program QSONG (Quasar Spectroscopic Observations with NIR Grism) has the feasibility to measure rest-frame optical $(\mathrm{H} \alpha$ or $\mathrm{H} \beta$ ) based $\mathrm{BH}$ masses at $\mathrm{z}>3$, which would be difficult from the ground due to atmospheric absorption and high sky background.

\section{SURVEY DESCRIPTION AND CURRENT STATUS}

Our high-z QSONG sample are $\sim 150$ luminous quasars at $3<\mathrm{z}<6$, out of SDSS (Schneider et al., 2007) and APM-UKST (Storrie-Lombardi et al., 1996, 2001) surveys. The targets are optically selected, spectroscopically confirmed type-I AGN, while flux-limited to zband $\mathrm{AB}$ magnitudes of $\sim 18.5$ and 19 in bright and faint subsamples, with more pointings of exposure time assigned to fainter targets for clear line detection. All targets were observed in near-infrared grism (NG) 


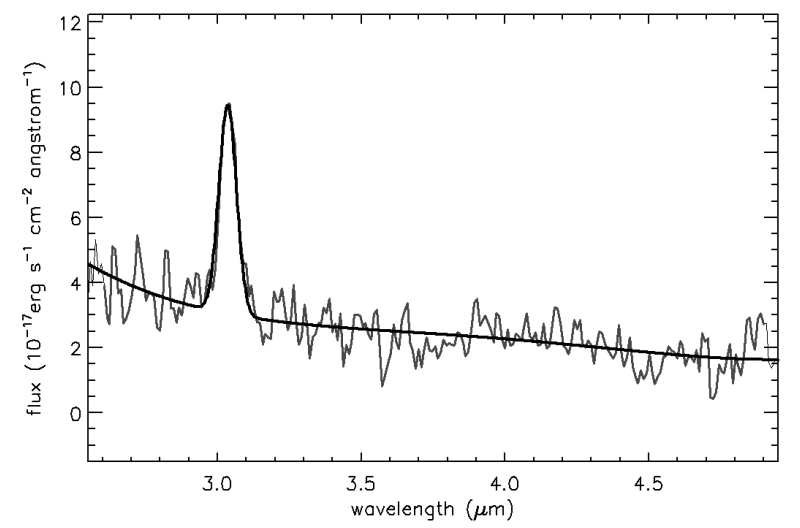

Fig. 1. An example of fitting a $z=3.6$ AKARI QSONG quasar spectrum, with continuum and emission line model components overplotted.

mode with spectral resolution of $\mathrm{R}=120$, while a limited number of additional prism $(\mathrm{R}=20)$ observations were performed to better investigate continuum properties.

Until the final observations in January 2010, the QSONG survey covered 762 pointings of quasars during its 1st and 2nd year of warm phase (phase 3 ) observations, where $\sim 60 \%$ of the data are targeted for high$\mathrm{z}$ quasars. Due to the relatively strong background noise in phase 3, careful data reduction was applied to obtain a cleaner set of spectra. After running the spectroscopic pipeline (IRC_SPEC_TOOLKIT_P3 version 20090211) we subtracted remaining hot pixels using L.A.Cosmic (van Dokkum, 2001), and stacked multiply observed pointings with sigma clipping applied over wavelength matched spectra. We checked the use of the treatment above, and found the $\mathrm{H} \alpha$ emission feature to stay in its shape while the continuum got cleaner. Selecting objects that meet meaningful S/N for emission line analysis ( $>5$ within the vicinity of the $\mathrm{H} \alpha$ emission line), we measured $\mathrm{BH}$ masses using the $\mathrm{H} \alpha$ based mass estimator (Greene \& Ho, 2005) after fitting the spectra around the emission line (Fig 1).

\section{SCIENCE TOPICS}

Having obtained a set of rest-frame optical BH mass estimates in high-z, we summarize ongoing research topics.

- Comparison of $\mathrm{H} \alpha$ and CIV based BH masses: this expands previous studies on the usefulness of UV based $\mathrm{BH}$ masses to higher redshift.
- Mass distribution of luminous high-z quasars: depending on the results from the topic above, we are to better understand the growth of BHs in the early universe $(z>5)$ with more reliable $\mathrm{BH}$ masses out of rest-frame optical and/or UV emission lines.

\section{ACKNOWLEDGEMENTS}

This work was supported by the the Creative Research Initiative program, No. 2010-0000712, of the National Research Foundation of Korea (NRFK) funded by the Korea government (MEST).

\section{REFERENCES}

Greene, J. E. \& Ho, L. C., 2005, Estimating Black Hole Masses in Active Galaxies Using the $\mathrm{H} \alpha$ Emission Line, ApJ, 630, 122

McConnell, N. J., et al., 2011, Two Ten-Billion-SolarMass Black Holes at the Centres of Giant Elliptical Galaxies, Nature, 480, 217

Mortlock, D. J., et al., 2011, A Luminous Quasar at a Redshift of $\mathrm{z}=7.085$, Nature, 474,616

Onken, C. A., et al., 2004, Supermassive Black Holes in Active Galactic Nuclei. II. Calibration of the Black Hole Mass-Velocity Dispersion Relationship for Active Galactic Nuclei, ApJ, 615, 645

Schneider, D. P., et al., 2007, The Sloan Digital Sky Survey Quasar Catalog. IV. Fifth Data Release, AJ, 134, 102

Shen, Y. \& Liu, X., 2012, Comparing Single-epoch Virial Black Hole Mass Estimators for Luminous Quasars, ApJ, 753, 125

Storrie-Lombardi, L. J., et al., 1996, APM Z >=4 QSO Survey: Spectra and Intervening Absorption Systems, ApJ, 468, 121

Storrie-Lombardi, L. J., et al., 2001, The Second APM UKST Colour Survey for z $>4$ Quasars, MNRAS, 322, 933

van Dokkum, P. G., 2001, Cosmic-Ray Rejection by Laplacian Edge Detection, PASP, 113, 1420

Volonteri, M. \& Rees, M. J., 2005, Rapid Growth of High-Redshift Black Holes, ApJ, 633, 624 